\title{
Glaucoma in patients with uveitis
}

\author{
William C Panek, Gary N Holland, David A Lee, Robert E Christensen
}

\begin{abstract}
The records of 100 patients (161 eyes) with uveitis were reviewed retrospectively to determine the prevalence of increased intraocular pressure, the forms of uveitis most commonly associated with glaucoma, and the forms that require specific glaucoma therapy. Secondary glaucoma was present in 23 patients (31 eyes): three of 24 patients with acute uveitis (three eyes, $12 \%$ of acute uveitis patients) and 20 of 76 patients with chronic uveitis (28 eyes, $26 \%$ of chronic uveitis patients). Eighteen patients (26 eyes, $78 \%$ of glaucoma patients) with chronic uveitis required long-term medical therapy to control intraocular pressure. Three patients (three eyes, $12 \%$ of glaucoma patients) with acute uveitis required short-term therapy to control intraocular pressure. The remaining two patients had transient increases in intraocular pressure that did not require treatment or that responded to treatment of intraocular inflammation alone. One patient with chronic uveitis (two eyes, $4 \%$ of glaucoma patients) required filtering surgery. At least five patients (eight eyes, $22 \%$ of glaucoma patients) had glaucomatous visual field defects. The results of this survey are consistent with the concept that secondary glaucoma is a management problem in patients with anterior segment inflammation and chronic rather than acute uveitis. An algorithm for the management of increased intraocular pressure in patients with uveitis is proposed.
\end{abstract}

Particularly complex management decisions arise when intraocular inflammation is coupled with increased intraocular pressure. The delicate tissues of the anterior segment may become swollen, scarred, and distorted, or rendered malfunctional by the inflammatory response, ${ }^{1}$ leading to increase of intraocular pressure, glaucomatous damage to the optic nerve, and subsequent loss of visual field. Steroids, the mainstay of treatment for uveitis, may also cause increased intraocular pressure. ${ }^{2}$ The physician must evaluate carefully the effects of both disease and therapy in order to provide proper management for patients with uveitis and glaucoma.

This study was undertaken to obtain data on the prevalence of increased intraocular pressure in patients with uveitis and to identify the forms of uveitis most commonly associated with glaucoma and that require specific glaucoma therapy. With such information rational approaches to the management of uveitis-related glaucoma can be planned.

Subjects and methods

The records of 100 consecutive patients with uveitis referred to and examined by one author (GNH) at the Jules Stein Eye Institute, UCLA School of Medicine, were reveiwed retrospectively. Uveitis was identified by the presence of inflammation involving any intraocular structure at the time of examination or by a well documented history of intraocular inflammation. Excluded were patients with self-limited anterior chamber inflammatory reactions during a onemonth period following intraocular surgery, patients with neoplastic disease mimicking uveitis ('masquerade syndromes'), and patients with cytomegalovirus (CMV) retinopathy. *

Each case was examined for location of inflammation, disease chronicity, and the presence or history of increased intraocular pressure. For the purpose of this study, the patients were considered to be glaucoma suspects or to have uveitic glaucoma if intraocular pressure was above $21 \mathrm{~mm} \mathrm{Hg}$ on more than one examination at UCLA, or if the patient was receiving antiglaucoma therapy at the time of initial examination at UCLA and previously had a well documented increase in intraocular pressure concurrent with intraocular inflammation. No patients had a history of preexisting primary open angle glaucoma.

The cases were divided into acute and chronic uveitis as defined by the International Uveitis Study Group. ${ }^{3}$ Cases of acute uveitis were less than three months in duration and cases of chronic uveitis were of three months or more.

\section{Results}

There were 161 eyes with uveitis among the 100 patients investigated in this study (Table 1). Twenty-four patients ( 34 eyes) had acute uveitis and 76 (127 eyes) had chronic uveitis. All the patients had cells and flare in the anterior chamber, indicating anterior segment inflammation, regardless of the primary site of disease. Of the patients with acute uveitis all had primary inflammation of the anterior segment except three, who had toxoplasmic retinochoroiditis with secondary iridocyclitis. Eleven patients with chronic uveitis had intermediate uveitis (pars planitis). Of the remaining 65 patients with chronic uveitis, one with VogtKoyanagi-Harada syndrome, one of three with sarcoidosis, and one of 33 with uveitis of unknown cause had inflammation of the posterior segment; all others had inflammation of the anterior segment alone.

Twenty-three patients ( 31 eyes) had secondary uveitic glaucoma by our criteria. Three patients (three eyes) had acute uveitis and 20 patients ( 28 eyes) had chronic uveitis. Glaucoma occurred in

^The author examines a large number of patients with CMV retinopathy enrolled in clinical research protocols. They would have made up a disproportionately large percentage of the consecutive uveitis cases had they been included. No patient with CMV retinopathy had an increased intraocular pressure. 
TABLE I Patients with uveitis and increased intraocular pressure (IOP)

\begin{tabular}{|c|c|c|c|c|}
\hline Diagnosis & $\begin{array}{l}\text { Number of } \\
\text { patients }\end{array}$ & $\begin{array}{l}\text { Total eyes } \\
\text { with } \\
\text { inflammation }\end{array}$ & $\begin{array}{l}\text { Patients with } \\
\text { increased IOP }\end{array}$ & $\begin{array}{l}\text { Total eyes } \\
\text { with } \\
\text { increased IOP }\end{array}$ \\
\hline $\begin{array}{l}\text { Acute uveitis } \\
\text { HLA B27+ } \\
\text { HLA B27- } \\
\text { Toxoplasmosis } \\
\text { Posner-Schlossman syndrome }\end{array}$ & $\begin{array}{r}11 \\
9 \\
3 \\
1\end{array}$ & $\begin{array}{r}15 \\
13 \\
5 \\
1\end{array}$ & $\begin{array}{l}0 \\
0 \\
2 \\
1\end{array}$ & $\begin{array}{l}0 \\
0 \\
2 \\
1\end{array}$ \\
\hline Total & 24 & 34 & 3 & 3 \\
\hline $\begin{array}{l}\text { Chronic uveitis } \\
\text { Unknown cause } \\
\text { Intermediate (pars planitis) } \\
\text { Juvenile rheumatoid arthritis } \\
\text { Intraocular lens-associated } \\
\text { Fuchs' heterochromic iridocyclitis } \\
\text { Herpes simplex virus associated } \\
\text { Sarcoidosis } \\
\text { Syphilis } \\
\text { Herpes zoster virus associated } \\
\text { Vogt-Koyanagi-Harada syndrome }\end{array}$ & $\begin{array}{r}33 \\
11 \\
7 \\
6 \\
5 \\
5 \\
3 \\
3 \\
2 \\
1\end{array}$ & $\begin{array}{r}61 \\
20 \\
14 \\
6 \\
6 \\
5 \\
6 \\
5 \\
2 \\
2\end{array}$ & $\begin{array}{l}8 \\
1 \\
3 \\
2 \\
2 \\
2 \\
1 \\
0 \\
1 \\
0\end{array}$ & $\begin{array}{r}13 \\
1 \\
5 \\
2 \\
2 \\
2 \\
2 \\
0 \\
1 \\
0\end{array}$ \\
\hline Total & 76 & 127 & 20 & 28 \\
\hline
\end{tabular}

$12 \%$ of patients with acute uveitis and in $26 \%$ of patients with chronic uveitis. There was no statistically significant difference in the incidence of glaucoma between these two groups. All 23 glaucoma patients had at some time undergone steroid therapy - oral, topical, or periocular. Treatment regimens at the time of this review varied from prednisone $60 \mathrm{mg}$ orally every day combined with prednisolone acetate $1 \%$ solution every hour to fluorometholone $1 \%$ solution every other day. Only one patient with glaucoma was off steroid therapy. No cases of glaucoma were shown to be secondary to steroid response.

Toxoplasmic retinochoroiditis was the only form of posterior uveitis in which secondary anterior segment inflammation resulted in increased intraocular pressure. PosnerSchlossman syndrome was the only acute primary anterior segment disorder that was found to be associated with increased intraocular pressure. All the other patients had chronic uveitis, either primary anterior segment inflammation alone or panuveitis. All the patients with bilateral uveitis who developed glaucoma did so in both inflamed eyes. At least five patients (eight eyes, $22 \%$ of glaucoma patients) had glaucomatous visual field defects.

Twenty-one patients ( 29 eyes, $91 \%$ of glaucoma patients) received antiglaucoma medications for control of intraocular pressure. Eighteen patients ( 26 eyes, $78 \%$ of glaucoma patients) required continuous, ongoing therapy; all had chronic uveitis. The other three patients (three eyes, $13 \%$ of glaucoma patients), all of whom had acute uveitis, required brief medical therapy only during the initial period of inflammation. The remaining two patients with glaucoma (two eyes, $9 \%$ of glaucoma patients), both of whom had chronic anterior uveitis, did not require medical therapy; they had histories of transient, self-limited increases in intraocular pressure that responded to treatment of inflammation alone. One patient (two eyes, 4\% of glaucoma patients) with chronic uveitis required filtering surgery to lower the intraocular pressure.

Discussion

The spectrum of uveitic disorders in this series is consistent with that seen in other referral practices. ${ }^{4}$ Uveitis may be complicated by a variety of secondary problems including cataract formation, synechiae, macular oedema, and glaucoma. In the present series glaucoma was one of the most common complications that required therapeutic intervention.

The factors most commonly associated with problems in the management of glaucoma were disease chronicity and primary anterior segment inflammation. Toxoplasmic retinochoroiditis was the only disorder in which secondary anterior segment inflammation resulted in rises of intraocular pressure requiring medical therapy. Increased intraocular pressure associated with acute uveitis was not a significant management problem, generally responding well to brief periods of medical therapy. Glaucoma associated with chronic uveitis usually required prolonged medical therapy for control of pressure. Even with chronic uveitis, however, treatment of inflammation alone brought intraocular pressure under control in some cases. Visual field loss was a problem only in patients with chronic uveitis.

In this group of referral patients it is possible that the incidence of raised intraocular pressure among patients with acute uveitis was underestimated. A transient rise in pressure associated with the onset of intense inflammation that resolved with the initiation of anti-inflammatory therapy by referring physicians may have been missed. It is unlikely, however, that such transient increases in pressure pose a serious problem to the eye.

\section{PATHOPHYSIOLOGY OF UVEITIC}

\section{GLAUCOMA}

The status of the anterior chamber angle gives an important anatomical division into either closed or open angle types of glaucoma, and has important therapeutic and prognostic implications. In patients with uveitis the angle can be closed as a result of posterior synechiae producing pupillary seclusion and iris bombé. ${ }^{5}$ Angle closure also can occur without pupillary block. It can result from peripheral anterior synechiae (an iridotrabecular adherenceassociated inflammation of iris and angle 
structures) and sometimes inflammatory nodules in the angle. ${ }^{16}$ In addition rubeosis iridis leading to fibrovascular scarring and closure of the angle can occur in patients with uveitis. ${ }^{7}$ In this series no cases of angle closure were seen, indicating that this mechanism may occur infrequently in patients receiving antiinflammatory therapy.

Increased intraocular pressure associated with a clinically open angle involves microscopical changes with the trabecular meshwork. Postulated mechanisms include (1) mechanical blockage of the trabeculum by serum components that are liberated because of vascular incompetence; $^{8}$ (2) hypersecretion associated with prostaglandin mediated vascular hyperpermeability; ${ }^{10}$ (3) overtaxing of outflow mechanisms by protein that interferes with active transport; ${ }^{11}$ (4) inflammation of the trabeculum itself with swelling that causes impaired outflow; ${ }^{6}$ (5) damage to trabecular endothelial cells by the inflammatory process; ${ }^{12}$ (6) mechanical obstruction of outflow by precipitates on the meshwork; ${ }^{13}$ (7) sclerosis of trabecular meshwork as a result of chronic inflammation; 5 or (8) obstruction of the trabeculum by a hyaline membrane. ${ }^{5}$

Steroid therapy is also a potential cause of raised pressure, ${ }^{214} 15$ though in the present series no cases of steroid induced glaucoma were recorded. Steroid induced glaucoma is very similar to primary open angle glaucoma, ${ }^{16}$ occurring slowly over weeks to months. Occasionally, however, an acute increase in pressure is noted. In all cases the trabeculum is clinically normal in appearance. Cessation of steroids usually leads to a return to normal of the pressure. ${ }^{16}$
In many patients with anterior segment inflammation who are receiving chronic steroid therapy it is not clear whether increased intraocular pressure is due to the effect of the inflammation or steroids. There is not always a relationship between the amount of inflammation, as measured by cells and flare, and inflammation induced rise of intraocular pressure. One therefore cannot predict in which patients intraocular pressure will drop when steroid therapy is increased. The authors have seen patients with only $1+$ cells in the anterior chamber who had a dramatic drop in pressure with an increased dosage of steroids.

\section{GLAUCOMA ASSOCIATED WITH SPECIFIC UVEITIC DISORDERS}

Many different types of uveitis have been associated with glaucoma, but certain disorders may have a relatively higher risk. For example, glaucoma is believed to be the major long term threat to vision in patients with Fuchs' heterochromic iridocyclitis. ${ }^{17-19}$ Though frequently intermittent and well controlled initially, glaucoma may become chronic and difficult to treat.

Glaucoma is also a major component of the Posner-Schlossman syndrome (glaucomatocyclitic crisis). ${ }^{20}$ Though inflammatory signs may be minimal, rises in intraocular pressure may be severe, leading to blurred vision, haloes, and ocular discomfort. Pressures of 40-50 $\mathrm{mmHg}$ are not uncommon. Raitta and Vannas believe that there may be a relationship between this syndrome and the eventual development of primary open angle glaucoma. ${ }^{21}$

Segmental iris ischaemia, demonstrated on

\section{Management of Intraocular Pressure in Patients with Uveitis}

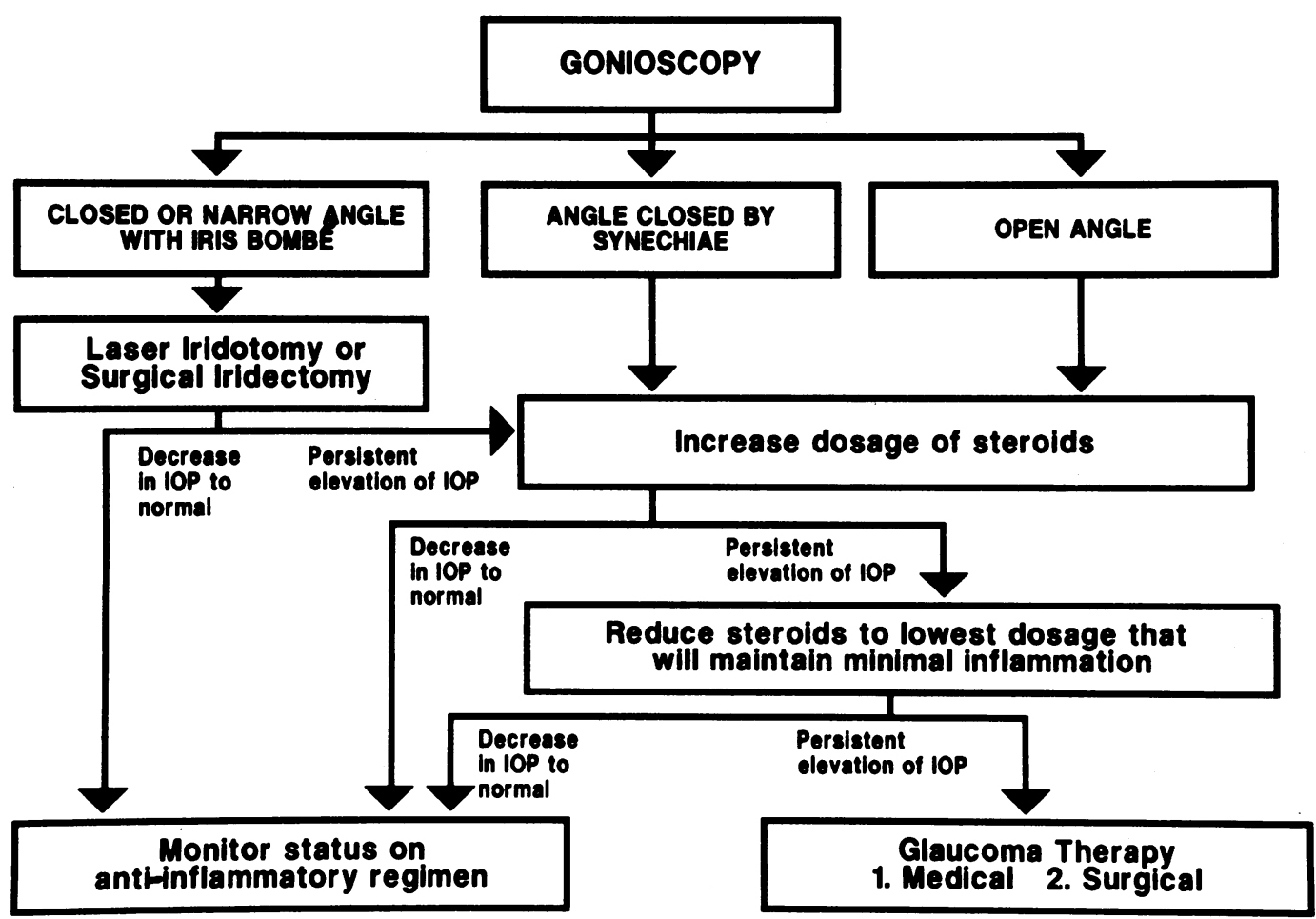

Figure 1: Algorithm for the management of raised intraocular pressure in patients with uveitis. 
fluorescein angiography, implies a vascular cause or the Posner-Schlossman syndrome. ${ }^{21}$ Vasculature incompetence could be associated with a release of prostaglandins, inflammation and a subsequent rise in intraocular pressure. ${ }^{22}$ Oral indomethacin and subconjunctival polyphloretin, both prostaglandin inhibitors, have been shown to lower intraocular pressures during attacks. ${ }^{22}$

The prognosis for control of intraocular pressure in patients with glaucomatocyclitic crisis is good. ${ }^{23}$ Current treatment recommendations include both topical steroid therapy to reduce inflammation and antiglaucoma medications to control pressure during acute attacks. Future modes may include antiprostaglandin therapy as well as treatment to modify vascular instability. ${ }^{21}$

The chronic uveitis associated with systemic disease is frequently complicated by severe glaucoma. ${ }^{24}$ The glaucoma associated with juvenile rheumatoid arthritis commonly occurs with open angles but may be of the pupillary block, secondary angle closure type, as a result of the formation of posterior synechiae. ${ }^{25} 26$ When glaucoma occurs in patients with sarcoid uveitis, medical and surgical therapy may have only limited success. ${ }^{27}$

Glaucoma may be a prominent feature of the uveitis that results from ocular infections. The prevalence of secondary glaucoma associated with toxoplasmic retinochoroiditis was reported to be $12 \%$ in one series. ${ }^{29}$ Although the glaucoma in that group was described as transient, a more recalcitrant form may occur with severe infections.

Herpetic uveitis may be particularly prone to the development of glaucoma. ${ }^{30}$ In severe cases long term antiglaucoma therapy may be indicated even after active inflammation has subsided ${ }^{30}$ Herpes simplex virus keratouveitis is often associated with a raised intraocular pressure; Falcon and Williams reported glaucoma in $28 \%$ of 183 patients..$^{30}$ Postulated mechanisms include both blockage of the trabeculum with inflammatory debris and inflammation of the trabeculum. ${ }^{31}$ Townsend and Kaufman found that glaucoma was also a problem in experimental herpes simplex virus keratouveitis in the rabbit. ${ }^{32}$ Secondary glaucoma was attributed to a mononuclear cellular infiltrate in the angle; in addition occasional peripheral anterior synechiae were seen in severe cases.

Secondary glaucoma also can be a serious problem in patients with herpes zoster virus keratouveitis; Womack and Liesegang reported uveitic glaucoma in 10 of 37 patients. ${ }^{33}$ The pathophysiology is probably similar to that associated with herpes simplex virus keratouveitis. ${ }^{30}$

Uveitis may complicate the use of iris supported or anterior chamber intraocular lenses, ${ }^{34}$ more likely as a result of mechanical factors such as chafing of the lens against ocular tissues. The smouldering low-grade uveitis may lead to secondary glaucoma; Ellingson described a distinct syndrome of uveitis, glaucoma, and hyphaema in such patients. ${ }^{35}$ The prognosis for the control of pressure is good if inflammation can be controlled.
EVALUATION AND TREATMENT OF UVEITIC GLAUCOMA

The treatment of intraocular inflammation itself is an important component of glaucoma management, because it prevents damage to angle structures and blockage of outflow channels by inflammatory material and thereby maintains normal aqueous outflow. ${ }^{36}$ In one case of the present series the control of inflammation by chlorambucil in a patient having a 20 -year history of uveitis associated with juvenile rheumatoid arthritis also resulted in lowering of intraocular pressure that could not be controlled by antiglaucoma medications alone.

There may be special considerations in the medical management of glaucoma in patients with uveitis. Miotics such as pilocarpine should be used with caution in inflamed eyes, because they may exacerbate the underlying inflammatory process. ${ }^{36}$ The ocular hypotensive effect of epinephrine is inhibited by cyclo-oxygenase inhibitors such as indomethacin. Patients using epinephrine should be monitored for this pressure-raising effect in those rare cases in which non-steroidal anti-inflammatory agents are used in the management of uveitis. ${ }^{37}$

The laser and surgical treatment of glaucoma in patients with uveitis also involves special considerations. Laser iridotomy may be difficult to perform in inflamed eyes ${ }^{36}$ and may cause scarring. Should surgical peripheral iridectomy be required in cases of uveitis for the treatment of pupillary block glaucoma, surgical section or large peripheral iridectomies should be considered. ${ }^{36}$ The efficacy of laser trabeculoplasty varies in these patients ${ }^{38}$ and may lead to a worsening of both inflammation and glaucoma.

For patients with chronic inflammatory glaucoma some authors ${ }^{36}$ recommend trabeculodialysis as the procedure of choice. Success rates as high as $63 \%$ with this technique have been reported, in contrast to rates of $18 \%$ with standard filtration surgery. ${ }^{39}$

Adjunctive methods of treatment are under investigation to decrease postoperative inflammation and fibrosis after glaucoma filtering surgery. They include the use of locally administered agents such as 5-fluorouracil ${ }^{40}$ and triamcinolone ${ }^{+1}$ in the preoperative and postoperative periods. In future these regimens may be shown to improve filter survival in patients with uveitic glaucoma.

On the basis of these concepts the authors propose a stepwise approach to the evaluation and treatment of increased intraocular pressure in patients with uveitis (Fig 1). Because steroid associated glaucoma is apparently uncommon, and because the effect of prolonged or increased inflammation is potentially worse than a transient steroid induced rise in intraocular pressure, one should first increase steroid dosage and observe both intraocular pressure and inflammation. In cases where severe glaucomatous nerve damage previously exists, antiglaucoma therapy may be included while steroids are increased and then withdrawn if pressure decreases.

If intraocular pressure remains unchanged or rises with increased steroids, and inflammation is stable or improved, steroid therapy should be 
reduced to the least amount that will maintain the lowest achievable level of inflammation. If intraocular pressure remains raised as steroid therapy is reduced, glaucoma therapy is initiated. While the modes of therapy may be dictated by the presence of inflammation, the intensity of treatment - as with all forms of glaucoma - is based on the level of intraocular pressure, visual field changes, and status of the optic nerve.

Dr Panek was an Abe Meyer Fellow in Glaucoma, UCLA Department of Ophthalmology. Dr Holland is recipient of the Research to Prevent Blindness - James S Adams Scholar Award. The work was supported in part by a Research Manpower Award from Research to Prevent Blindness, Inc. New York, NY (D Holland), and Karl Kirchgessner Foundation, an unrestricted grant from Research to Prevent Blindness, Inc., Nationa Institutes of Health Grant EY07701, and the Lucille Ellis Simon Glaucoma Research Fund (Dr Lee), and the Elsie B Ballantyne Fund (Dr Christensen).

1 Ritch R. Pathophysiology of glaucoma in uveitis. Trans Ophthalmol Soc UK 1981; 101: 321-4.

2 David DS, Berkowitz JS. Ocular effects of topical and systemic corticosteroids. Lancet 1969; i: 149-51.

3 Bloch-Michel E, Nussenblatt RB. International study group recommendations for the evaluation of intraocular .

4 Henderly DE, Genstler AJ, Smith RE, Rao NA. Changing patterns of uveitis. Am 7 O phthalmol 1987; 103: 131-6.

5 Schlaegel TF. Complications of uveitis. Int Ophthalmol Clin 1977; 17: 65-74.

6 Mapstone $R$. Vascular factors in the aetiology of secondary glaucoma. Trans Ophthalmol Soc UK 1971; 91: 741-8.

7 Gartner S, Henkind P. Neovascularization of the iris (rubeosis iridis). Surv Ophthalmol 1978; 22: 291-312.

8 Epstein DL, Hashimoto JM, Grant WM. Serum obstruction of aqueous outflow in enucleated eyes. Am $\mathcal{F}$ Ophthalmol 1978; 86: 101-5.

9 Kass MA, Podos SM, Moses RA, Becker B. Prostaglandin E1 and aqueous humor dynamics. Invest Ophthalmol Vis $S c i$ and aqueous hum

10 Beitch BR, Eakins KE. The effects of prostaglandins on the intraocular pressure of the rabbit. Brf Pharmacol 1969; 37 intraocular

11 Zirm M. Protein glaucoma - overtaxing of flow mechanisms? Preliminary report. Ophthalmologica 1982; 184: 155-61.

12 Sherwood M, Richardson TM. Evidence for in vivo phagocytosis by trabecular endothelial cells. Invest Ophthalmol Vis Sci 1980; 21 (suppl): 66

13 Roth M, Simmons RJ. Glaucoma associated with precipitates on the trabecular meshwork. Ophthalmology 1979; 86: 16138 .

14 Armaly MF. Effects of corticosteroids on intraocular pressure and fluid dynamics. Arch Ophthalmol 1963; 70: 482-91.

15 Becker B, Mills D. Corticosteroids and intraocular pressure. Arch Ophthalmol 1963; 70: 500-7.

16 François J. Corticosteroid glaucoma. Ann Ophthalmol 1977; 9 1075-80
17 Lownenfeld IE, Thompson SH. Fuchs' heterochromic cyclitis: a critical review of the literature. Surv Ophthalmol $1973 ; 17: 394-414$

18 Kimura SJ, Hogan MJ, Thygeson P. Fuchs' syndrome of heterochromic cyclitis. Arch Ophthalmol 1955; 54; 179-86.

9 Liesegang T. Clinical features and prognosis in Fuchs' uveitis syndrome. Arch Ophthalmol 1982; 100: 1622-6.

20 Posner A, Schlossman A. Syndrome of unilateral recurrent attacks of glaucoma with cyclitic symptoms. Arch Ophthalmol 1948; 39: 517-35.

21 Raitta C, Vannas A. Glaucomatocyclitic crisis. Arch Ophthalmol 1977; 95: 608-12

22 Matsuda K, Izawa Y, Mishima S. Prostaglandins and glaucomatocyclitic crisis. $\mathcal{F p n} 7$ Ophthalmol 1975; 19: 36875 .

23 Hung PT, Chang JM. Treatment of glaucomatocyclitic crisis. Am F Ophthalmol 1974; 77: 169-72.

24 Krupin T, Dorfman NH, Spector SM, Wax MB. Secondary glaucoma associated with uveitis. Glaucoma $1988 ; 10: 85-90$.

$25 \mathrm{Key} \mathrm{SN} \mathrm{III,} \mathrm{Kimura} \mathrm{SJ.} \mathrm{Iridocyclitis} \mathrm{associated} \mathrm{with} \mathrm{juvenile}$ rheumatoid arthritis. Am f Ophthalmol 1975; 80: 425-9.

26 Kanski JJ. Anterior uveitis in juvenile rheumatoid arthritis. Arch Ophthalmol 1977; 95: 1794-7.

27 Hoover D, Khan J, Giagiacoma J. Pediatic ocular sarcoidosis. Surv Ophthalmol 1986; 30: 225-7.

28 Obenaut CD, Shaw HE, Sydnar CF, Klintworth GK Sarcoidosis and its ophthalmic manifestations. Am $\dot{f}$ Ophthalmol 1978; 86: 648-55.

29 Friedmann CT, Knox DL. Variations in recurrent active toxoplasmic retinochoroiditis. Arch Ophthalmol 1969; 81: 481-93.

30 Falcon MG, Williams HP. Herpes simplex keratouveitis and glaucoma. Trans Ophthalmol Soc UK 1978; 98: 101-4.

31 Hogan MJ, Kimura SF, Thygeson P. Pathology of herpes simplex kerato-iritis. Am f Ophthalmol 1964; 57: 551.

32 Townsend WM, Kaufman HE. Pathogenesis of glaucoma and endothelial changes in herpetic keratouveitis in rabbits. Am 7 Ophthalmol 1971; 71: 904-10.

33 Womack LW, Liesegang TJ. Complications of herpes zoster ophthalmicus. Arch Ophthalmol 1983; 101: 42-5.

34 Layden WE. Pseudophakia and glaucoma. Ophthalmology 1982; 89: 875-9.

35 Ellingson FT. The uveitis-glaucoma-hyphema syndrome associated with the Mark VII Choyce anterior chamber lens implant. 7 Am Intraocul Implant Soc 1978; 4: 50-3.

36 Hoskins HD, Hetherington J, Shjaffer RN. Surgical management of the inflammatory glaucomas. Perspect Ophthalmol 1977; 1: 173-81.

37 Camras CB, Feldman SG, Podos SM, Christensen RE, Gardner SK, Fazio DT. Inhibition of the epinephrineinduced reduction of intraocular pressure by systemic indomethacin in humans. Am $\mathcal{F}$ Ophthalmol 1985; 100: 169-75.

38 Robin AL, Pollack IP. Argon laser trabeculoplasty in the secondary glaucomas. Int Congr Ophthalmol 1982; 1: 620.

39 Kanski JJ, Shun-Shin GA. Systemic uveitis syndromes in childhood: an analysis of 340 cases. Ophthalmology 1984; 91 : 1247-51.

40 Heuer DK, Parrish RK, Gressel MG, et al. 5-Fluorouracil and glaucoma filtering surgery. Intermediate follow-up of a pilot glaucoma filtering surgery. Intermediate
study. Ophthalmology 1986; $93: 1537-46$.

41 Giangiacomo J, Dueker D, Adelstein EA. The effect of preoperative subconjunctival triamcinolone administration of glaucoma filtration. Trabeculectomy following subconjunctival triamcinolone. Arch Ophthalmol 1986; 104: 83841 . 